Original Article

\title{
Giant cerebellopontine Angle Tumor Surgery: Experience of a Tertiary Care Center in Lahore, Pakistan
}

\author{
Tariq Imran Khokhar, Anam Fatima, Sumaira Kiran, Muhammad Naveed Majeed \\ Hafiz Abdul Majid, Asif Bashir \\ Department of Neurosurgery Unit III, Punjab Institute of Neurosciences, Lahore
}

\begin{abstract}
Objectives: In this case series, we report our experience of microsurgical resection of large and giant CPA tumors at the Department of Neurosurgery, Punjab Institute of Neurosciences (PINS).

Materials and Methods: This was a retrospective case series of 328 patients (mean age, 40 years) with large and giant CPA tumors (predominantly vestibular schwannomas) who underwent surgical removal using a retro sigmoid approach over 4 years.

Results: In the study, there were $58 \%$ (190) females while $42 \%$ (138) were males. $60 \%$ (197) of the tumors were right - sided and $40 \%$ (131) left - sided. Hearing loss was the main presenting complaint with $73 \%$ of the patients having non-serviceable hearing. There were 14 (4.3\%) deaths reported during the retrospective analytic study. There were 3 cases of postoperative hemorrhage, and 11 patients expired due to postoperative wound infection. There were 13 cases diagnosed as having post-operative bacterial meningitis. Gross total tumor excision was achieved in $98 \%$ of patients based on postoperative imaging. There were 26 cases (8\%) with postoperative CSF leakage that was managed with LP drain (5 patients) and in the rest VP shunt was done. Facial nerve function was graded according to the House Brackmann system. It was recorded in all patients following surgery: There were $16 \%$ patients with HB grade IV and 216 (66\%) patients with HB grade III facial palsy. Other complications included 52 (16\%) cases of physiologic injury to lower cranial nerves needing post-operative tracheostomy and PEG for $4-6$ weeks.
\end{abstract}

Conclusion: Suboccipital Retrosigmoid approach is ideal for dealing with giant CPA tumors. Complication rates in our series were comparable with other reported literature.

Keywords: Cerebellopontine angle, Retrosigmoid, Giant CPA.

Corresponding Author: Tariq Imran Khokhar

Department of Neurosurgery Unit III

Punjab Institute of Neurosciences (PINS), Lahore

Email: nsdrkhokhar2014@gmail.com

Date of Submission: 01-12-2021

Date of Revision: 22-12-2021

Date of Acceptance: 21-12-2021
Date of Online Publishing: 31-12-2021

Date of Print: 31-12-2021

DOI: $10.36552 /$ pjns.v25i4.620

\section{INTRODUCTION}

The most common location for tumors in the 
posterior fossa is the CP angle. Nearly ten percent of all cerebral neoplasms are these tumors. ${ }^{1}$ Vestibular schwannoma (VS) is the most frequent CP angle tumor, accounting for $80-90$ percent of all cases. Meningiomas, which account for about $5-10 \%$ of all tumors, are the second most common. The epidermoid cyst is the third most frequent type of $\mathrm{CP}$ angle tumor, accounting for about 5-7 percent of all CP angle tumors. Metastatic lesions, arachnoid cysts, cranial nerve V, VII, IX, X, X neuromas, vascular malformations, aneurysms, cholesterol granulomas, and neurenteric cysts are just a few of the uncommon tumors that can appear in this area. ${ }^{2}$

CPA has complex anatomy with various important neurovascular structures traversing through it. Clinical manifestations of tumors in this region occur due to loss of function of the neural structures, as a result of direct compression. ${ }^{3}$ Vestibular schwannomas can present with unilateral hearing loss, other symptoms like tinnitus, vertigo, and gait disturbances can also be present. The incidence of VS among NF2 patients is higher than in the general population. Meningiomas are mostly large tumors at the time of diagnosis and patients clinically present with the dysfunction of cranial $\mathrm{V}$ $\& X$ i.e. trigeminal neuralgia, facial dysesthesias, facial numbness \& swallowing difficulties. Epidermoid cysts can also present with symptoms similar to that of VS and meningiomas. ${ }^{4}$ Treatment strategies for CPA tumors comprise wait and scan policy, microsurgical resection of the tumor, and gamma knife radiosurgery. ${ }^{5}$ Surgery of CPA tumors has always been a great challenge due to the complex anatomical structure of the area. However, advances in micro neurosurgical techniques, neuroanesthesia, and intraoperative neurophysiological monitoring have considerably reduced postoperative morbidity and mortality. CPA tumor surgery aims to achieve a complete tumor resection without damaging the hearing and facial nerve function and also to improve the patient's quality of life. ${ }^{6}$
There are various approaches for micro neurosurgical excision of these tumors, like translabyrinthine, suboccipital (retrosigmoid), transcochlear, transotic, and middle fossa craniotomies. The selection of the best approach to achieve maximum resection depends upon the pathologic entity, size of the tumor, and involvement of the adjacent structures. Surgical outcomes depend upon the approach chosen, size and location of the tumor and tumor histology. ${ }^{7}$ Retrosigmoid approach is the most versatile approach which provides excellent visualization of CPA, brainstem, and the internal auditory canal (IAC). The Translabyrinthine approach is good for early identification of facial nerves and better visualization of IAC. However, this approach can't be employed on patients with serviceable hearing. The middle fossa approach is suitable for tumors that are exclusively present in IAC as CPA visualization is poor through this approach. Gamma knife radiosurgery is most suitable for vestibular schwannomas upto $3 \mathrm{~cm}$ in cisternal diameter. It is not suitable for patients who present with profound clinical symptoms and mass effects. ${ }^{8}$ A lot of work has already been done on various aspects of CPA tumors. Numerous studies have been published on surgical outcomes. However, the majority of these studies included tumors with a mean size, not more than $20 \mathrm{~mm}$. Our case series includes cases with almost all tumors in size greater than $30 \mathrm{~mm}$. In this study, we are going to share our experience of these complex tumors, which will highlight the patterns of CPA tumors in our part of the world. Moreover, this study would also highlight the significance of the retro sigmoid approach in dealing with huge CPA tumors. Surgical outcomes like post-operative preservation of facial nerve, hearing status, mortality, and recurrence of the tumor would also be discussed. Our study would provide an insight on how to deal with large tumors through the retro sigmoid approach. 


\section{MATERIALS AND METHODS}

\section{Study Design}

This is a retrospective data analysis of case series of 328 patients with CPA tumors who were hospitalized at the Punjab Institute of Neurosciences Unit 3 between January 2017 and September 2021.

\section{Inclusion Criteria}

All patients of both genders (mean age, 40 years) with large and giant CPA tumors (predominantly vestibular schwannomas) underwent surgical removal using retro sigmoid approach over 4 years.

\section{Exclusion Criteria}

Pediatric population.

\section{Data Collection Procedure}

Preoperative MRI was performed on all patients for radiological diagnosis. Patients were chosen based on their age, tumor size, hearing status before surgery, general medical condition, and surgical expectations. A single surgeon operated on all of the patients. We primarily used the suboccipital retro sigmoid method, but we also used the Translabyrinthine technique in a few individuals. Each patient's age, gender, hearing, and facial nerve status before and after surgery, tumor size, and postoperative complications were all collected. According to the INI or Samii grading system, tumors were graded based on radiography. T1: Intrameatal tumor, T2: Intra-extra metal tumor, T3: Intra-extrameatal tumor T3a: Filling the cerebellopontine cistern, T3b: Reaching the brainstem, T4a: Compressing the brainstem, T4b: Distorting the brainstem and compressing the fourth ventricle Pure tone audiometry before surgery. PTA (Post-traumatic amnesia) was used to determine the severity of hearing loss. SRT (Speech Reception Threshold) greater than $50 \mathrm{~dB}$ and SDS (Speech Discrimination Score) less than $50 \%$ were used to indicate unserviceable hearing. The facial nerve function was graded using the House Brackmann Scale. The connections between tumor size and resection extent, complication rate, reoperation necessity, and KPS were investigated. The effects of surgical technique and tumor size on pre-and postoperative HBS, KPS at discharge, and histology report were investigated.

\section{RESULTS}

\section{Age and Gender Distribution}

A total of 328 patients were included in the study. The mean age of the patients was 40 years with a range of $20-55$ years. There was a slight female predominance with 190 (58\%) being females and $138(42 \%)$ males.

\section{Laterality of Tumors}

$60 \%$ (197) of the tumors were right-sided and 40\% (131) were left - sided (Table 1).

\section{Tumor Size}

The mean tumor size was $40 \mathrm{~mm}$ with a range of $25 \mathrm{~mm}$ to $55 \mathrm{~mm}$. $66 \%$ of tumors were grade IV, $16 \%$ were grade $V$ and $18 \%$ were grade III tumors. $2(0.6 \%)$ recurrent cases, previously operated outside our hospital were done.

\section{Frequent Complaints}

The most frequent complaints were sensorineural hearing loss. $73 \%$ (239) cases had non-serviceable hearing pre-operatively. $11.6 \%$ (38) patients had absent corneal reflexes and hypoesthesias. $16 \%$ of patients had basal cranial nerves involvement. In $35 \%$ (115) cases preoperatively VP shunt was done (Table 2). 


\section{Surgical Approach}

6 (2\%) cases were operated through the Translabyrinthine approach and 321 (98\%) via the suboccipital retrosigmoid approach (Table 4).

Table 1: Laterality of Tumors.

\begin{tabular}{lcc|} 
Parameters & No. of Patients & Percentage \\
Right sided & 197 & $60 \%$ \\
Left Sided & 131 & $40 \%$ \\
\hline
\end{tabular}

Table 2: Presenting Complaints.

\begin{tabular}{lcc|} 
Parameters & No of Patients & Percentage \\
Tinnitus & 207 & $63 \%$ \\
Vertigo & 138 & $42 \%$ \\
Hearing Loss & 236 & $72 \%$ \\
Facial Nerve Palsy & 219 & $67 \%$ \\
Trigeminal Nerve & 36 & $11 \%$ \\
Involvement & & \\
Basal Cranial Nerve & 46 & $14 \%$ \\
Involvement & & \\
\hline
\end{tabular}

Table 3: Serviceable Hearing.

\begin{tabular}{lcc} 
Parameters & No of Patients & Percentage \\
Serviceable Hearing & 89 & $27 \%$ \\
Non-Serviceable & 239 & $73 \%$ \\
Hearing & & \\
\hline
\end{tabular}

Table 4: Surgical Approach.

\begin{tabular}{lcc|} 
Parameters & No. of Patients & Percentage \\
Retrosigmoid & 321 & $98 \%$ \\
Translabyrinthine & 6 & $2 \%$ \\
\hline
\end{tabular}

\section{Histopathology}

Pathology in 230 (70\%) cases were vestibular schwannomas. There were $16(5 \%)$ cases of fifth nerve neuroma, 7 (2.2\%) cases of basal cranial nerves schwannomas. There were 59 (18\%) cases with the pathological diagnosis of meningiomas, 11 (3.3\%) epidermoid tumors, and 5 (1.5\%) cases of metastatic CA with all patients being male (Table 6). All the images are shared in results after the patient's consent.
Table 5: Tumor Resection.

\begin{tabular}{lcc|} 
Parameters & No. of Patients & Percentage \\
Gross Total & 321 & $98 \%$ \\
Sub-Total & 6 & $2.00 \%$ \\
\hline
\end{tabular}

Table 6: Histopathology.

$\begin{array}{lcc}\text { Parameters } & \text { No of Patients } & \text { Percentage } \\ \text { Vestibular Schwanoma } & 230 & 70 \% \\ \text { Meningioma } & 59 & 18 \% \\ \text { Epidermoid } & 11 & 3 \% \\ \text { Metastatic } & 05 & 2 \% \\ \text { 5th Nerve Neuroma } & 16 & 5 \% \\ \text { 9, 10, 11, 12 Nerve } & 07 & 2 \% \\ \text { Schwanoma } & & \end{array}$

Table 7: Complications.

$\begin{array}{lcc}\text { Parameters } & \text { No of Patients } & \text { Percentage } \\ \text { Mortality } & 14 & 4 \% \\ \text { Basal Cranial Nerve Injury } & 52 & 16 \% \\ \text { 7th Nerve Injury } & 65 & 20 \% \\ \text { CSF Leakage } & 26 & 8 \% \\ \text { Meningitis } & 13 & 4 \% \\ \text { VP Shunting } & 20 & 6 \%\end{array}$

Table 8: Distribution of tumor Resections.

\begin{tabular}{lcc} 
Type & Number & Percentage \\
Gross Total & 321 & $98 \%$ \\
Sub Total & 07 & $2 \%$ \\
\hline
\end{tabular}

Table 9: Distribution of tumor Category.

\begin{tabular}{lcc} 
Tumor & Number & Percentage \\
Vestibular & 230 & $70 \%$ \\
Schwannoma & 59 & $18 \%$ \\
Meningioma & 11 & $3.3 \%$ \\
Epidermoid & 05 & $1 \%$ \\
Metastatic Lesion & 16 & $05 \%$ \\
Fifth CN' Neuroma & 07 & $2.2 \%$ \\
$9^{\text {th }}, 10^{\text {th }}, 11^{\text {th }}$ and $12^{\text {th }}$ & 07 \\
CN' Schwannoma & $\mathbf{3 2 8}$ & $\mathbf{1 0 0} \%$ \\
Total & \\
\hline
\end{tabular}

Following surgical outcomes were noted in this case series.

http//www.pakjns.org 


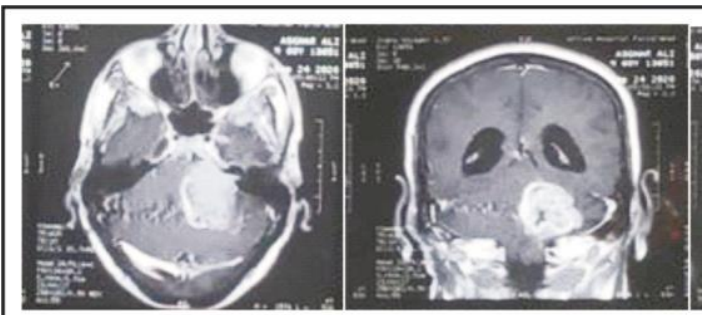

(a) Pre-op

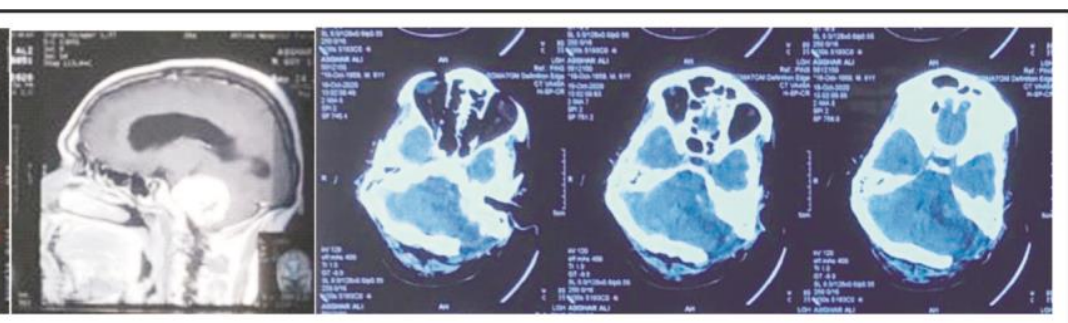

(b) Post-op

Fig 1: Vestibular Schwannoma.

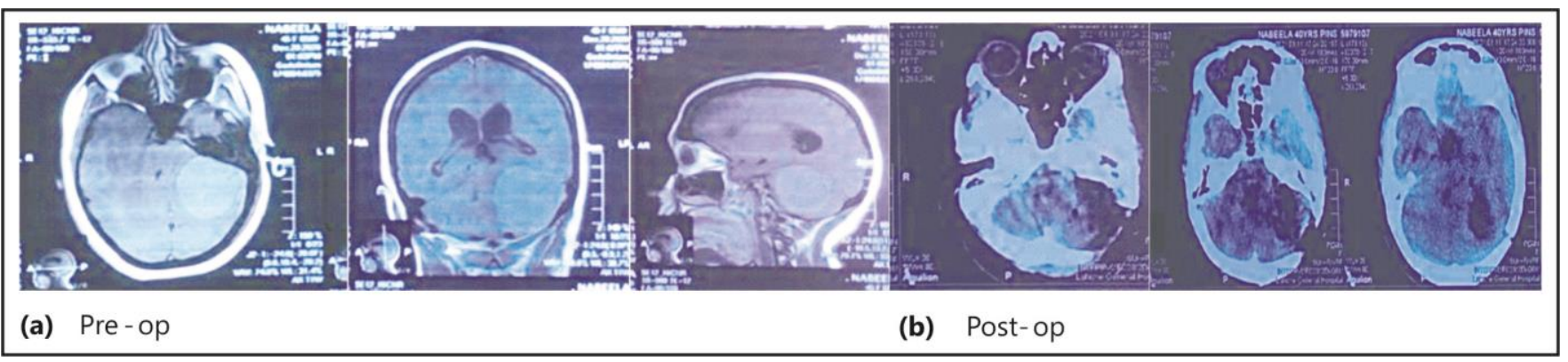

Fig 2: Meningioma.

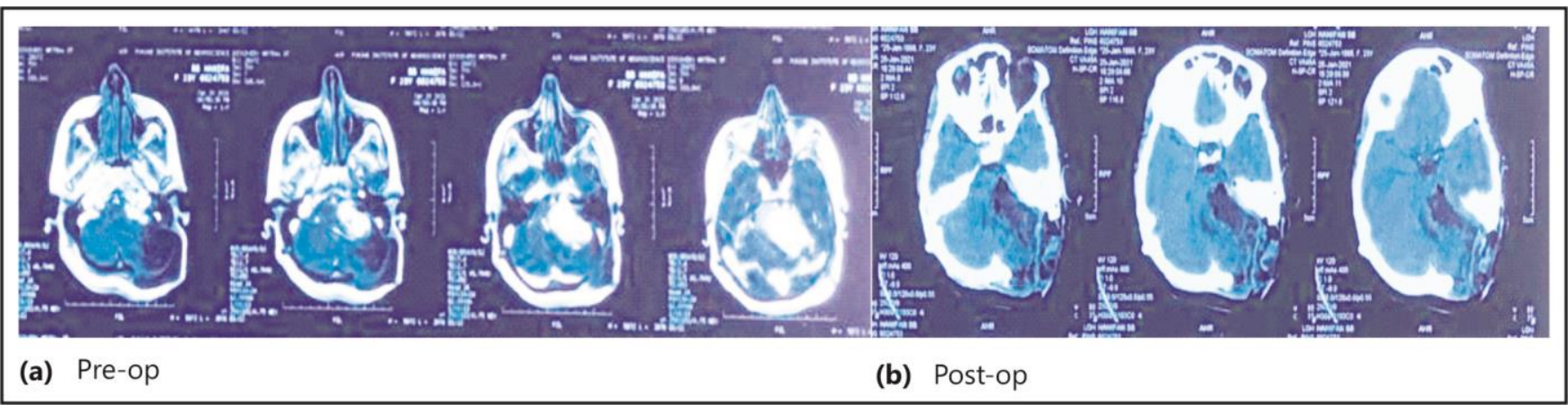

Fig 3: Residual tumor with Pseudomeningocele.

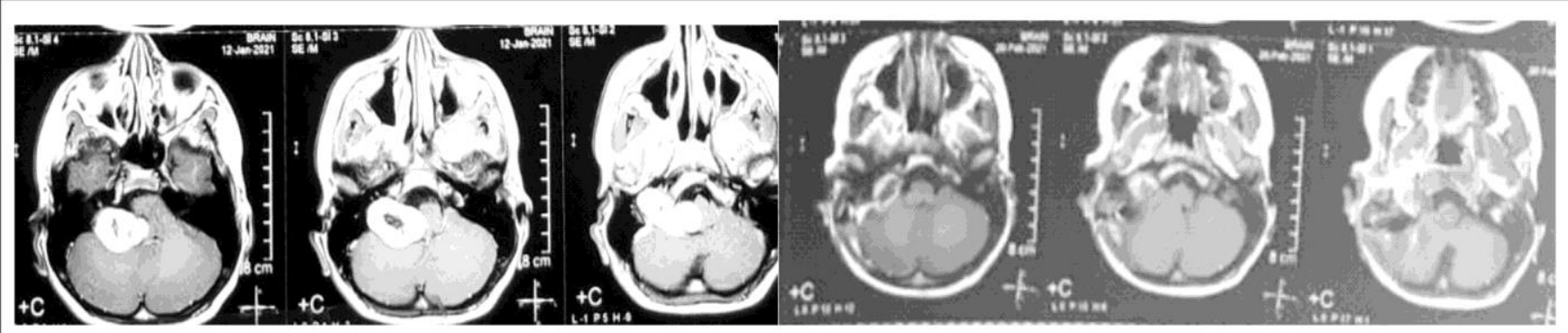

(a) Pre-op

(b) Post-op

Fig 4: $9^{\text {th }}$ CN Schwannoma. 


\section{Mortality}

There wasa total of 14 deaths (4.3\%) in this series, 3 were due to intracranial hemorrhage and 11 were due to post-operative wound infections (Table 7).

\section{Neurologic Complications (Excluding Facial Nerve Injury)}

We had almost 52 (16\%) patients of grade $v$ tumors. All these patients had a physiologic injury to basal cranial nerves $(9,10,11,12)$ per operatively. WE did tracheostomy and got their Percutaneous Endoscopic Gastrostomy (PEG) done postoperatively, which remained in place for $4-6$ weeks.

\section{Facial Nerve Function}

The House Brackmann scale was used to grade facial nerve function in the initial postoperative period. After surgery, all grade $\mathrm{V}$ tumors (16\%) had a House Brackmann scale of IV. The House Brackmann scale was III after surgery in $66 \%$ of grade IV tumors. All of our patients scored in the moderate range on the House Brackmann scale, i.e. (III \& IV).

\section{Cerebrospinal Fluid (CSF) Leakage}

There were 26 cases (8\%) with postoperative CSF leakage (Table 7). Five of them were managed with lumbar drain insertion and for the rest of the patients a medium pressure VP shunt was inserted.

\section{Meningitis}

There were 13 (4\%) cases diagnosed to have post-operative bacterial meningitis based on classic clinical symptoms and spinal fluid analysis (Table 7). All these patients were managed with Intravenous antibiotics, patients recovered without any further sequel.

\section{Residual Tumor}

Gross total resection was achieved in $98 \%$ percent of the cases (Table 5). There was no tumor recurrence in all of these patients on follow-up CT brain and MRI brain with IV contrast done within one year time period.

\section{DISCUSSION}

Because of the complicated neurovascular structures present in this area, operating on malignancies in the cerebellopontine angle is difficult. However, there has been a significant improvement in the treatment of malignant cancers. Since the early 1960 s, the introduction of microscopes and intraoperative monitoring tools for facial and vestibular nerve functions have transformed the surgical treatment of these malignancies. Our study has the most cases completed in a short period (4.8 years), which is greater than any other case series published in this part of the world. 10 The findings of 328 instances performed by a single surgeon are described in the study, which eliminates the complicating aspect of having many surgeons. The choice of surgical approach is mostly influenced by surgeons' preferences and their level of expertise. The suboccipital-retro sigmoid approach is the most commonly used approach for vestibular schwannoma and other CPA tumors, especially, whenever hearing preservation surgery is the main goal. It's also suggested for tumors of any size, regardless of hearing loss. Translabyrinthine surgery would allow for the safe removal of the tumor with fewer risks and an equally favorable outcome for the facial nerve when the goal is not to preserve hearing. We employed the suboccipital retro sigmoid route as our major technique in our study, with just a few instances being operated on using the translabyrinthine approach.

Our study strengthens the fact that the Suboccipital retrosigmoid approach is one of the best approaches for microsurgical resection. This 
fact is validated in several case series including the Majid Samii case series of 1000 patients of vestibular schwannomas. ${ }^{11}$ In our case series' most common presenting symptom was unilateral hearing loss, as the majority of the patients had vestibular schwannomas. The second most common presentation was lower motor neuron type facial palsy as around $82 \%$ of the tumors that were operated on were of grade IV \& V. In comparison to our study several other case series reported that tinnitus and vertigo were also among the main presenting complaints, however, our results were different in this regard. ${ }^{12}$

Complete tumor resection and facial and vestibulocochlear nerves preservation without further increasing the morbidity and mortality of the patients are considered to be the most important goal of CPA tumors surgery. In 98 percent of the cases, we were able to achieve the gross total resection, subtotal resection was done in the rest of the $2 \%$ which was due to tumor adherence to the brainstem or cranial nerves.

There were around $82 \%$ of the tumors with greater than $3 \mathrm{~cm}$ size in diameter and $73 \%$ of the patients had non-serviceable hearing, vestibular nerve preservation was not our primary goal. Facial nerve grade 2 and 3 involvement was already present in the majority of our patients. Despite the lack of availability of facial nerve monitoring in our setup, in 70 percent of the cases, anatomical preservation of facial nerve was achieved. ${ }^{13,14,15}$ our study demonstrates that size of the tumor and facial nerve preservation are directly correlated. The outcome of the facial nerve is worse when the tumors are larger. As compared to our study Majid Samii et al, in their study of 1000 vestibular schwannoma patients reported anatomical preservation of $93 \%$. However, in a study by Joarder et al facial nerve preservation was $74 \%$ for the large size tumors and $62 \%$ for giant tumors, which is comparable to our results. ${ }^{16}$ In our case series, $16 \%$ of patients had a physiological injury to caudal cranial nerves (9, 10, 11, 12) for which post-operative tracheostomy and PEG were done for $4-6$ weeks. This again emphasizes the fact that large and giant tumors have greater involvement of the nerves as compared to small tumors. Basal cranial nerves involvement can complicate the postoperative course of the patient, hence preoperative planning regarding feeding and tracheostomy are mandatory in large-sized tumors. In literature $1.5-5.5 \%$ rate of involvement of these nerves have been mentioned which is far less than our studies. This difference can be due to giant size tumors included in our study as compared to other studies.

Postoperative complications developed in our patients includes CSF leakage (8\%) from wound and meningitis (4\%). CSF leakage was managed through lumbar drainage and a few cases required post-operative VP shunt. Tumor size and type of surgical approach are the two main factors in the development of CSF leakage as concluded in several case series. The retrosigmoid approach has the highest frequency of CSF leakage as compared to the middle fossa approach. However, the rate has declined from $20 \%$ to almost $6 \%$ by the use of methods (fat, bone wax) to occlude mastoid air cells and obliteration of Eustachian tube. Also, preoperative shunting probably plays a role in decreased postoperative CSF leakage. ${ }^{19,20,21}$

Mortality was $4.3 \%$ which is high as compared to the series with a similar number of patients. Other studies have mentioned a rate of $1-5 \%$ mortality. Hence, the mortality rate is comparable with other series. However; mortality can be further reduced by proper pre-operative planning and post-operative care. In our series' mortality was due to post-operative wound infection and only two cases had an intracerebral hemorrhage.

\section{CONCLUSION}

Suboccipital retrosigmoid craniectomy is the best approach for the microsurgical excision of large 
and giant $\mathrm{CP}$ angle tumors. The size of the tumors has a great impact on the facial nerve and hearing preservation.CSF leakage and post-operative complications also seem to have an association with tumor size. Better results can be obtained through facial nerve monitoring; hence it is used must be employed to improve the quality of life of the patient. Earlier detection and intervention can preserve the major neurovascular structures and Improve the outcomes in terms of morbidity and mortality.

\section{REFERENCES}

1. Jawad MS, Alshalgi A, Abd A. Cerebellopontine Angle Tumors; Tumor Size-Outcome Relationship. Iraqi Academic Scientific Journal, 2018; 17 (2): 1527.

2. Moosa S, Ding D. Role of stereotactic radiosurgery in the management of Cerebellopontine angle tumors. Austin J Radiat. Oncol\& Cancer, 2015; 1 (1): 1004.

3. Broomfield SJ, O'Donoghue GM. Self-reported symptoms and patient experience: a British Acoustic Neuroma Association survey. British Journal of Neurosurgery, 2016 May 3; 30 (3): 294301.

4. Kentala E, Pyykkö I. Clinical picture of vestibular schwannoma. Auris Nasus Larynx. 2001 Jan. 1; 28 (1): 15-22.

5. Iwai Y, Yamanaka K, Ishiguro T. Surgery combined with radiosurgery of large acoustic neuromas. Surgical Neurology, 2003 Apr. 1; 59 (4): 283-9.

6. Memari F, Hassannia F, Abtahi SH. Surgical outcomes of cerebellopontine angle tumors in 50 cases. Iranian Journal of Otorhinolaryngology, 2015 Jan; 27 (78): 29.

7. Mazzoni A, Biroli F, Foresti C, Signorelli A, Sortino C, ZanolettiE. Hearing preservation surgery in acoustic neuroma. Slow progress and new strategies. Acta Otorhinolaryngol Ital. 2011; 31: 7684.22.

8. Samii M, Matthies C. Management of 1000 vestibular schwannomas (acoustic neuromas): surgical management and results with an emphasis on complications and how to avoid them. Neurosurgery, 1997; 40 (1): 11-21, Discussion 21-
3.27.

9. Falcioni $M$, Fois $P$, Taibah A, Sanna M. Facial nerve functionafter vestibular schwannoma surgery. J Neurosurg. 2011; 115: 820-6.29.

10. Awan MS, Qureshi HU, Sheikh AA, Ali MM. Vestibular schwannomas: clinical presentation, management and outcome. JPMA. The Journal of the Pakistan Medical Association, 2001; 51 (2): 63.

11. Samii M, Matthies C. Management of 1000 vestibular schwannomas (acoustic neuromas): surgical management and results with an emphasis on complications and how to avoid them. Neurosurgery, 1997 Jan. 1; 40 (1): 11-23.

12. Rauniyar S, Gu J, Wang L, Shen Z, Meng Q. Cerebellopontine Angle Tumors: Surgical and Non-Surgical Outcomes in a Cohort 321 Cases. Acta Scientific Neurology, 2020 Jun; 3 (6): (ISSN: 2582-1121).

13. Starnoni D, Giammattei L, Cossu G, Link MJ, Roche PH, Chacko AG, Ohata K, Samii M, Suri A, Bruneau $M$, Cornelius JF. Surgical management for large vestibular schwannomas: a systematic review, meta-analysis, and consensus statement on behalf of the EANS skull base section. Acta Neurochirurgica. 2020 Jul. 29: 1-23.

14. Nakamura $M$, Roser F, Dormiani M, Matthies C, Vorkapic P, Samii M. Facial and cochlear nerve function after surgery of cerebellopontine angle meningiomas. Neurosurgery, 2005 Jul. 1; 57 (1): 77-90.

15. Samii M, Gerganov VM, Samii A. Functional outcome after complete surgical removal of giant vestibular schwannomas. Journal of Neurosurgery, 2010 Apr. 1; 112 (4): 860-7.

16. Joarder $M A$, Karim $A B$, Sujon $S I$, Akhter $N$, Waheed-uz-Zaman $M$, Joseph $V$, Jahangir SM, Chandy MJ. Surgical outcomes of cerebellopontine angle tumors in 34 cases. Pulse, 2015; 8 (1): 8-

17. Ebersold MJ, Harner SG, Beatty CW, Harper CM, Quast LM. Current results of the retrosigmoid approach to acoustic neurinoma. Journal of Neurosurgery, 1992 Jun. 1; 76 (6): 901-9.

18. Lanman TH, Brackmann DE, Hitselberger WE, Subin B. Report of 190 consecutive cases of large acoustic tumors (vestibular schwannoma) removed via the translabyrinthine approach. Journal of Neurosurgery, 1999 Apr. 1; 90 (4): 617-23.

19. Slattery III WH, Francis $S$, House KC. Perioperative 
morbidity of acoustic neuroma surgery. Otology \&Neurotology, 2001 Nov. 1; 22 (6): 895-902.

20. Brennan JW, Rowed DW, Nedzelski JM, Chen JM. Cerebrospinal fluid leak after acoustic neuroma surgery: influence of tumor size and surgical approach on incidence and response to treatment. Journal of Neurosurgery, 2001 Feb. 1; 94 (2): 217-
23.

21. Becker SS, Jackler RK, Pitts LH. Cerebrospinal fluid leak after acoustic neuroma surgery: a comparison of the translabyrinthine, middle fossa, and retrosigmoid approaches. Otology \& Neurotology, 2003 Jan. 1; 24 (1): 107-12.

\section{Additional Information}

Disclosures: Authors report no conflict of interest.

Ethical Review Board Approval: The study was conformed to the ethical review board requirements.

Human Subjects: Consent was obtained by all patients/participants in this study.

Conflicts of Interest:

In compliance with the ICMJE uniform disclosure form, all authors declare the following:

Financial Relationships: All authors have declared that they have no financial relationships at present or within the previous three years with any organizations that might have an interest in the submitted work.

Other Relationships: All authors have declared that there are no other relationships or activities that could appear to have influenced the submitted work.

\section{AUTHORS CONTRIBUTIONS}

\begin{tabular}{|l|l|l|}
\hline Sr.\# & Author's Full Name & Intellectual Contribution to Paper in Terms of: \\
\hline 1. & Tariq Imran Khokhar & $\begin{array}{l}\text { 1. Study design, methodologies paper writing, referencing and data } \\
\text { calculations }\end{array}$ \\
\hline 2. & Anam Fatima & 2. Data Collection and Calculations. \\
\hline 3. & Sumaira Kiran & 3. Analysis of data and interpretation of results etc. \\
\hline 4. & Muhammad Naveed Majeed & 4. Literature review and manuscript writing. \\
\hline 5. & Tariq Imran Khokhar & 5. Analysis of data. \\
\hline 6. & Hafiz Abdul Majid & 6. Literature review. \\
\hline 7. & Asif Bashir & 7. Quality insurer. \\
\hline
\end{tabular}

